\title{
Maternal Plasma Betaine in Middle Pregnancy Was Associated with Decreased Risk of GDM in Twin Pregnancy: A Cohort Study
}

\author{
Xiaoli Gong' \\ Yufeng $\mathrm{Du}^{2}$ \\ Xiaona $\mathrm{Li}^{3}$ \\ Jing Yang' \\ Xinyuan Zhang' \\ Yuan Wei ${ }^{1}$ \\ Yangyu Zhao' \\ 'Department of Obstetrics and \\ Gynecology, National Clinical Research \\ Center for Obstetrics and Gynecology, \\ National Center for Healthcare Quality \\ Management in Obstetrics, Peking \\ University Third Hospital, Beijing, \\ I00191, People's Republic of China; \\ ${ }^{2}$ Department of Epidemiology and \\ Statistics, School of Public Health, \\ Lanzhou University, Lanzhou, 730000, \\ People's Republic of China; ${ }^{3}$ Department \\ of Pharmacy, Peking University Third \\ Hospital, Beijing, 100191, People's \\ Republic of China
}

Purpose: Although previous studies have shown that choline-related metabolites in one carbon metabolism (OCM) were related to gestational diabetes mellitus (GDM) risk in singleton pregnancy, their role in twin gestations remains unclear. We aimed to investigate the associations between choline, betaine, methionine, dimethylglycine (DMG), trimethylamine N-oxide (TMAO) and GDM risk among women with twin gestations.

Patients and Methods: This hospital-based cohort study included 187 women with dichorionic twin gestations. Blood samples were collected during pregnancy at a median of 16.1 weeks of gestation (IQR: 13.9 -17.9). Concentrations of plasma metabolites were measured by HPLC-triple quadrupole MS. Log-binomial regression models were applied to estimate the associations between plasma metabolites and the risk of GDM.

Results: A total of 57 (30.5\%) GDM cases were diagnosed over the study follow-up. Eightyseven percent of women conceived through ART. Plasma betaine had an inverse association with GDM risk, and the adjusted RR of GDM comparing the highest tertile with the lowest tertile was $0.41\left(95 \%\right.$ CI: $\left.0.19-0.86, P_{\text {trend }}=0.015\right)$. Women with a high betaine/choline ratio or a low DMG/betaine ratio were at decreased GDM risk $\left(P_{\text {trend }}=0.031\right.$ or 0.001 , respectively). Plasma choline, methionine, DMG and TMAO were not associated with GDM risk.

Conclusion: Among women with dichorionic twin gestations, higher plasma level of betaine in the second trimester was associated with lower risk of GDM. This finding needs further confirmation.

Keywords: one-carbon metabolism, betaine, gestational diabetes mellitus, twin pregnancy

\section{Introduction}

Gestational diabetes mellitus (GDM), one of the most common complications of pregnancy, is estimated to affect approximately $16.9 \%$ of pregnancies worldwide. ${ }^{1}$ Factors such as pre-pregnant body weight and body mass index (BMI), ${ }^{2}$ advanced age $^{3}$ and dietary patterns ${ }^{4}$ are associated with the prevalence of GDM. It is not only associated with adverse pregnancy outcomes including macrosomia, fetal dystocia, and cesarean delivery but also with increased diabetes risk for both mother and child in their later life, ${ }^{5,6}$ for example, children with intrauterine exposed to GDM have higher risk of adiposity. ${ }^{7}$ Twin gestations itself is an independent risk factor for GDM due to increased insulin resistance. ${ }^{8}$ Although available evidence is limited, several studies have shown that GDM in twin pregnancy is associated with similar adverse maternal and neonatal outcomes than that in singleton pregnancy. $^{9-11}$
Correspondence: Yangyu Zhao; Yuan Wei Email zhaoyangyu00I@I63.com; weiyuanbysy@163.com 
Choline is an essential nutrient necessary for the integrity of cell membranes, cholinergic neurotransmission and one-carbon metabolism. ${ }^{12}$ In one-carbon metabolism, choline is irreversibly oxidized to betaine, which donates its methyl group in the remethylation of homocysteine to methionine, a reaction generating dimethylglycine $(\mathrm{DMG})^{13}$ (Figure 1). Trimethylamine N-oxide (TMAO) is a gut microbial-derived metabolite of choline, betaine and L-carnitine. Emerging evidence suggests that choline and its related metabolites may contribute to the pathogenesis of insulin resistance. ${ }^{14-16}$ Betaine supplementation has been shown to reduce fasting glucose and improve insulin resistance in mouse models. ${ }^{14,16}$ An animal study found that dietary TMAO increased fasting insulin levels and exacerbated impaired glucose tolerance. ${ }^{15} \mathrm{~A}$ metabonomic study in pregnant women showed that higher urine choline, lower plasma betaine and TMAO were associated with increased risk of GDM, respectively. ${ }^{17}$ So far, two epidemiologic studies have examined the association between choline and its related metabolites and GDM risk in singleton pregnancies. ${ }^{18,19}$ A U-shaped association of choline and an inverse association of betaine with GDM risk were reported in a nested case-control study. ${ }^{18}$ For TMAO, these two studies showed inconsistent results, one showed an inverse association but the other revealed a positive association with GDM risk. ${ }^{18,19}$ To the best of our knowledge,

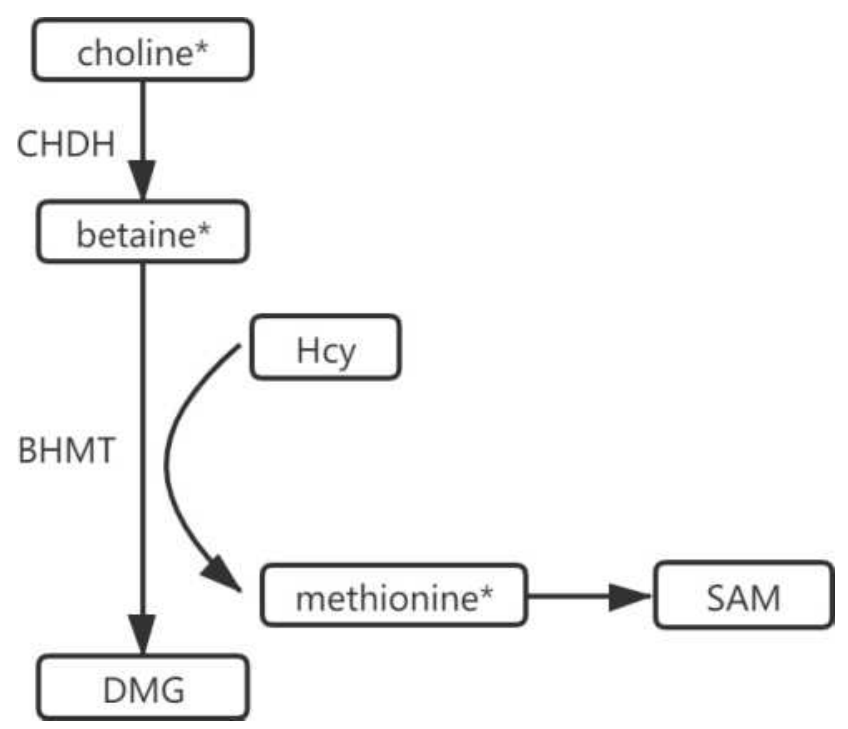

Figure I Choline oxidation metabolites in one carbon metabolism. *They can be obtained from dietary.

Abbreviations: $\mathrm{CHDH}$, choline dehydrogenase; DMG, dimethylglycine; Hcy, homocysteine; SAM, S-adenosylmethionine. no previous studies have explored the relationship between choline-related metabolites and GDM risk in twin pregnancy.

Considering the increasing number of twin pregnant women as a result of the widespread use of reproductive techniques and advanced maternal age and the increasing incidence of GDM worldwide, ${ }^{1,20}$ identifying the potential risk factors of GDM in this high-risk group would provide important public health implications. Our aim is to investigate the relationship between plasma concentrations of choline, betaine, methionine, DMG, TMAO and GDM risk in twin pregnancy in a hospital-based cohort. The association between the ratio of betaine to choline or DMG to betaine with GDM risk are also explored because the ratios were considered as stronger predictors of metabolic disturbances. ${ }^{21}$

\section{Methods}

\section{Study Participants}

A hospital-based cohort of women with twin pregnancy was established between March 2017 and December 2018 in Peking University Third Hospital in Beijing, China. Twin pregnant women who received regular antenatal care and planned to delivery at this hospital were recruited. Participants were excluded as follows: those who underwent fetal reduction, those who were pregnant with monochorionic-monoamniotic (MCMA) twins or monochorionic diamniotic (MCDA) twins, and those who presented chromosomal and other congenital abnormalities. A total of 314 women with twin gestations were recruited, 127 of whom were excluded due to the exclusion criteria, resulting in 187 women with dichorionic twin gestations included in the study eventually.

\section{Collection of Covariates}

When participants underwent the first antenatal care visit, nurses measured their height and the value was accurate to $0.01 \mathrm{~m}$. Pre-pregnancy BMI was calculated as prepregnancy weight ( $\mathrm{kg}$, self-reported data from women) divided by height squared $\left(\mathrm{m}^{2}\right)$. Gestational weight gain was calculated by subtracting the weight before pregnancy from the weight before delivery. Information on age at childbirth, ethnicity, occupation, parity, mode of conception, coexisted diseases (complications) and infant sex were extracted from questionnaires completed during antenatal care visits or medical records after admission.

Gestational age at birth was calculated using the last menstrual period and was further confirmed or revised by 
early ultrasound. Gestational age based on ultrasound findings was used when there was a discrepancy larger than seven days. For women used assisted reproduction techniques (ART) for conception, gestational age was calculated from embryo transfer day plus 17 or 19 days in pregnancy for cryopreserved embryos transfer pregnancy. Chorionicity was determined by sonographic findings (placental sites and lambda or T-signs) and was further confirmed by examination of the placenta after delivery.

\section{Diagnosis of GDM}

At 24 to 28 weeks of gestational age, DCDA women underwent the $75 \mathrm{~g} 2 \mathrm{~h}$ One Step test to identify GDM which was recommended by the International Association of the Diabetes and Pregnancy Study Groups (IADPSG) and the American Diabetes Association (ADA). ${ }^{22}$ Blood was drawn on an empty stomach in the morning; $75 \mathrm{~g}$ of glucose was then dissolved in 200 to $300 \mathrm{~mL}$ of water and drunk within five min. The time was recorded when the first mouthful of sugar water was drunk, and blood was drawn every hour. GDM was diagnosed if fasting plasma glucose was $\geq 5.1 \mathrm{mmol} / \mathrm{L}, 1$-h after oral glucose tolerance test (OGTT) plasma glucose was $\geq 10.0 \mathrm{mmol} / \mathrm{L}$ or 2 -h after OGTT plasma glucose was $\geq 8.5 \mathrm{mmol} / \mathrm{L}$. The median gestational age at OGTT test was 24 weeks (range: 22-28 weeks), and most women (91.9\%) took the OGTT test between 24 and 25 weeks.

\section{Blood Samples Collection and Preparation}

Early morning fasting blood samples were collected in vacuum blood tubes (EDTA-K2) with routine pregnancy check-ups, after centrifugation, plasma was separated and stored at $-80^{\circ} \mathrm{C}$ refrigerator until tested. All blood samples were collected before 23 weeks of gestation.

In brief, $50 \mu \mathrm{L}$ plasma was precipitated by adding 300 $\mu \mathrm{L}$ methanol containing internal standards (choline-d9, betaine-d3, DMG-d6, methionine- $\mathrm{d} 3$ and TMAO-d9). The mixture was further vortexed for 30 seconds and then centrifuged at $15,000 \mathrm{~g}$ for $15 \mathrm{~min}\left(4^{\circ} \mathrm{C}\right)$. The supernatant was analyzed by using HPLC-MS/MS analysis.

\section{Measurement of Plasma Metabolites}

We applied HPLC (Shimadzu)- Triple Quadrupole MS (API 4000+; AB Sciex, USA) to analyze the concentrations of plasma choline, betaine, DMG, methionine and TMAO. A XBridge BEH Amide column (1.7 $\mu \mathrm{m}$,
$2.1 \times 100 \mathrm{~mm}$, Waters, US) was used for metabolites separation. The column flow rate was $0.3 \mathrm{~mL} / \mathrm{min}$, and the sample injection volume was $1 \mu \mathrm{L}$. The column temperature was $35^{\circ} \mathrm{C}$. The mobile phases consist of phase A ( $0.1 \%$ formic acid in acetonitrile) and phase B (water with $0.1 \%$ formic acid and $5 \mathrm{mM}$ ammonium formate). The gradient program commenced at $15 \% \mathrm{~B}$ at $0.01 \mathrm{~min}$, then slowly increased to $45 \% \mathrm{~B}$ at $8.5 \mathrm{~min}$, followed by increased to $80 \% \mathrm{~B}$ at $8.5 \mathrm{~min}$ and maintained for $2 \mathrm{~min}$. The targeted metabolites and their internal standards were monitored positive ion and in multiple reaction monitoring (MRM) mode. MS parameters were optimized for each metabolite and internal standard. The inter-day CVs of quality control samples (at the low, medium and high concentration) during analyses were as follows: $1.5 \%$ $-7.4 \%$ for choline, $2.8-5.6 \%$ for betaine, $3.7 \%-6.5 \%$ for DMG, $2.2 \%-5.2 \%$ for methionine, $2.2-5.9 \%$ for TMAO. All sample measurements were conducted by LCMS technicians who were blinded to GDM status of our participants.

\section{Statistical Analysis}

Concentrations of all metabolites were presented as medians with interquartile ranges (IQRs) because of non-normal distributions. Differences of participant characteristics and plasma metabolite concentrations between women with and without GDM were compared using Mann-Whitney $U$-test or $t$ test or Chi-square test as appropriate.

We tested nonlinear associations between plasma metabolites and GDM risk using restricted cubic spline models with three knots at the 10th, 50th, and 90th centiles, and no nonlinear relationship was detected (all $\mathrm{P}_{\text {nonlinearity }}>0.1$ ). Since odds ratios often overestimate risk ratios when the occurrence of outcome events is not rare, ${ }^{23}$ we applied log-binomial regression models to directly estimate relative risks (RRs) of GDM in relation to plasma metabolites given the high GDM rate (30.5\%) in our study. Robust Poisson regression models were used if log-binomial models did not converge. A log-transformation was performed to improve the normality of metabolites distributions, and a standard deviation score (SDS) was then created for each of the metabolites before further analyses. The effect size is interpreted as changes in GDM risk associated with per SD change of log-transformed metabolite concentration. Besides, Concentrations of plasma metabolites and ratios of metabolites were categorized into tertiles based on the cutoffs defined among all participants. RRs were estimated from models with adjustment for pre-pregnancy BMI, age, 
parity, ART, gestational age at blood sampling, ethnicity, occupation, infant sex, hypertensive disorders in pregnancy, gestational weight gain (Model 1) and from models adjusted for covariates in Model 1 plus the five mutually adjusted plasma metabolites (Model 2). All participants were not smokers and were not drinking during pregnancy in our study. Thus, smoking and drinking were not considered in multivariable models. Mutual adjustment of each metabolite was conducted to obtain the independent association of each metabolite with GDM risk. $P_{\text {trend }}$ was obtained by entering the categorized tertiles into a regression model as a continuous variable.

To explore the potential modification effect by prepregnancy BMI, age, parity or infant sex for GDM, stratified analyses were conducted. $P_{\text {interaction }}$ was obtained by adding multiplicative interaction terms in the multivariable models. We performed sensitivity analyses by assessing the association between metabolite and GDM risk after excluding ethnic minorities or women conceived naturally. All statistical tests were 2-tailed and $P$ values $<0.05$ were considered as significant. Statistical analyses were performed by SPSS software (version 24.0) and R statistical software (version 3.4.0).

\section{Results}

The characteristics of study population were showed in Table 1. Among 187 participants, 57 developed GDM during the follow-up. The majority of mothers were Han Chinese (94.1\%), white-collar workers $(69 \%)$, nulliparous (78.1\%), and were conceived by ART (87.2\%). Nearly half of mothers were older than 35 years old $(44.9 \%)$. The incidence of hypertensive disorders in pregnancy was $26.7 \%$. Women of advanced reproductive age were more common in GDM group than non-GDM group (56.1\% vs $40.0 \%, P=0.041$ ), whereas all other characteristics were similar among two groups. For plasma concentration of metabolites, the median betaine concentration was significantly lower in GDM group compared with the non-GDM group $(13.8 \mu \mathrm{mol} / 1$ vs $15.3 \mathrm{umol} / 1, P=0.023)$.

We investigated the associations between metabolites and GDM risk. Plasma betaine had an inverse association with GDM risk in all three models, and stronger association was observed with the inclusion of more variables in regression models (Table 2). In the fully adjusted model, the RRs of GDM with the increase of plasma betaine tertiles were 1.00 (ref.), 0.54 (95\% CI: $0.28,1.06)$ and 0.41 (95\% CI: $0.19,0.86)$, respectively $\left(P_{\text {trend }}=0.015\right)$. Each SD increase in betaine concentration was associated lower risk of GDM with an RR of 0.68 (95\% CI 0.47 , 1.00). Plasma choline, DMG, methionine or TMAO were not related to GDM risk in all three models.

Furthermore, we also examined the ratio of upstream and downstream metabolites of betaine (Table 3). The RR of GDM for the highest (vs lowest) tertile of the plasma betaine: choline ratio was 0.48 (95\% CI: $\left.0.25,0.91, P_{\text {trend }}=0.031\right)$ in the adjusted model. Notably, with regard to the plasma DMG:betaine ratio, highest tertile was selected as reference group. Women in the lowest tertile of DMG:betaine ratio had lower risk of GDM compared with the highest tertile (RR: 0.42 95\% CI:0.24, 0.74, $\left.P_{\text {trend }}=0.001\right)$ in the adjusted model.

The reference model included traditional risk factors produced a receiver operating characteristic curve (ROC) area under the curve (AUC) of 0.67 (Table 4, Figure 2, Curve 1). The ROC AUC reached 0.74 after adding choline, DMG, methionine and TMAO in model 1 (Curve 2). After further adding betaine, the ROC AUC was significantly improved to 0.76 ( $P=0.042$ vs the reference model). Model which additionally included betaine: choline and DMG: betaine performed best in predicting GDM risk (AUC $=0.79, P=0.006$ vs the reference model).

The association between betaine and GDM risk was not modified by pre-pregnancy BMI, age, parity or infant sex; consistent associations were observed in each strata of these risk factors and all $P$ values for interaction were not statistically significantly (data were not shown). In sensitivity analyses after excluding ethnic minorities or women conceived naturally, the relationship between plasma betaine and GDM risk still existed although the sample sizes were much smaller (data were not shown).

\section{Discussion}

To our knowledge, this is the first study to investigate the associations between choline-related metabolites and GDM risk in twin pregnancy. We observed that plasma levels of betaine during the second trimester was negatively related to the risk of GDM. However, plasma choline, DMG, methionine and TMAO were not significantly associated. Besides, we found that high betaine: choline ratio or low DMG: betaine ratio, which contained upstream or downstream metabolites of betaine, was related to the decreased risk of GDM.

Our finding that plasma betaine was inversely associated with GDM risk was in line with findings from a nested case-control study among Chinese women with singleton pregnancy, ${ }^{18}$ a markedly elevated risk of GDM was observed for women with lower betaine status $(\leq 200$ 
Table I Characteristics of Mother and Child Pairs in This Cohort ${ }^{\mathrm{a}}$

\begin{tabular}{|c|c|c|c|c|}
\hline & Total $(n=187)$ & $\begin{array}{c}\text { Non-GDM } \\
(n=130)\end{array}$ & GDM (n=57) & $P$ value \\
\hline Age, n (\%), years & & & & 0.041 \\
\hline$<35$ & $103(55.1)$ & $78(60.0)$ & $25(43.9)$ & \\
\hline$\geq 35$ & $84(44.9)$ & $52(40.0)$ & $32(56.1)$ & \\
\hline Ethnicity, n (\%) & & & & 0.266 \\
\hline Han Chinese & $176(94.1)$ & $124(95.4)$ & $52(91.2)$ & \\
\hline Ethnic minorities & II (5.9) & $6(4.6)$ & $5(8.8)$ & \\
\hline Occupation, n (\%) & & & & 0.908 \\
\hline Administrator/other white-collar worker & $129(69.0)$ & $90(69.2)$ & $39(68.4)$ & \\
\hline Blue-collar worker & II (5.9) & $7(5.4)$ & $4(7.0)$ & \\
\hline Unemployed/freelancer/student/other & $47(25.1)$ & $33(25.4)$ & $14(24.6)$ & \\
\hline Pre-pregnancy BMI, n (\%), kg/m² & & & & 0.079 \\
\hline$<18.5$ & $13(7.0)$ & II (8.5) & $2(3.5)$ & \\
\hline $18.5-24.9$ & $124(66.3)$ & $90(69.2)$ & $34(59.6)$ & \\
\hline$\geq 25$ & $50(26.7)$ & $29(22.3)$ & $21(36.8)$ & \\
\hline Parity, n (\%) & & & & 0.849 \\
\hline Nulliparous & $146(78.1)$ & I0I (77.7) & 45 (78.9) & \\
\hline Multiparous & $4 \mid(21.9)$ & $29(22.3)$ & $12(21.1)$ & \\
\hline Mode of conception, $\mathbf{n}(\%)$ & & & & 0.881 \\
\hline ART & $163(87.2)$ & $113(86.9)$ & $50(87.7)$ & \\
\hline Spontaneous & $24(12.8)$ & $17(13.1)$ & $7(12.3)$ & \\
\hline Hypertensive disorders in pregnancy, n (\%) & & & & 0.177 \\
\hline Yes & $50(26.7)$ & $31(23.8)$ & $19(33.3)$ & \\
\hline No & $137(73.3)$ & $99(76.2)$ & $38(66.7)$ & \\
\hline Infant sex, $\mathrm{n}(\%)$ & & & & 0.148 \\
\hline Male-male & $62(33.2)$ & $35(26.9)$ & $8(14.0)$ & \\
\hline Female-female & $43(23.0)$ & $40(30.8)$ & $22(38.6)$ & \\
\hline Female-male & $82(43.9)$ & $55(42.3)$ & $27(47.4)$ & \\
\hline Gestational weight gain, mean (sd), kg & $15.0(5.1)$ & $16.5(5.4)$ & $15.3(4.4)$ & 0.126 \\
\hline Gestational age at blood sampling, median ( $95 \%$ range), weeks & I6.1 (12.6-20.7) & $16.2(12.6-20.3)$ & $16.0(12.4-21.3)$ & 0.509 \\
\hline \multicolumn{5}{|l|}{ Plasma concentrations, median (25th-75th percentile), $\mu \mathrm{mol} / \mathrm{I}$} \\
\hline Choline & $12.7(10.9,15.0)$ & $12.5(10.4,14.9)$ & $12.9(11.7,15.4)$ & 0.252 \\
\hline Betaine & $15.2(\mid 2.8,17.7)$ & $15.3(13.1,19.0)$ & $13.8(12.0,17.0)$ & 0.023 \\
\hline Dimethylglycine & $1.6(1.3,1.9)$ & $1.6(1.2,1.9)$ & $1.7(1.4,2.0)$ & 0.218 \\
\hline Methionine & $14.2(12.0,16.8)$ & $14.0(11.9,16.8)$ & $14.4(12.0,16.3)$ & 0.820 \\
\hline Trimethylamine- $\mathrm{N}$-oxide & $1.5(1.1,2.1)$ & $1.5(I .1,2.3)$ & I.4 (I.I, I.9) & 0.480 \\
\hline
\end{tabular}

Notes: ap values for differences of baseline characteristics and plasma metabolites concentration between pregnant women with and without GDM were obtained from Mann-Whitney U-test or $t$ test or Chi-square test.

Abbreviations: BMI, body mass index; ART, assisted reproductive technology; GDM, gestational diabetes mellitus.

$\mathrm{nmol} / \mathrm{mL}$ ) in this study (RR: 4.44, 95\% CI: 2.19, 9.00). A metabonomic study conducted in Portugal reported an inverse association of plasma betaine with GDM risk. ${ }^{17}$ A recent meta-analysis has shown that women with a history of GDM have a nearly 10 -fold increased risk of developing type 2 diabetes mellitus (T2DM). ${ }^{24} \mathrm{~T} 2 \mathrm{DM}$ and GDM share many of the same risk factors and pathogenesis. We are aware of several epidemiologic studies 
Table 2 Associations of Metabolites Concentrations with GDM Risk ${ }^{\mathrm{a}}$

\begin{tabular}{|c|c|c|c|c|}
\hline & \multirow[t]{2}{*}{ GDM/Subtotal } & Unadjusted & Model I & Model 2 \\
\hline & & $\operatorname{RR}(95 \% \mathrm{CI})$ & $\operatorname{RR}(95 \% \mathrm{CI})$ & RR (95\% Cl) \\
\hline \multicolumn{5}{|l|}{ Choline } \\
\hline Tertile I & $13 / 62$ & 1.00 (ref.) & 1.00 (ref.) & 1.00 (ref.) \\
\hline Tertile2 & $26 / 63$ & $1.97(1.11,3.47)$ & I.8I $(0.92,3.54)$ & I.8I $(0.88,3.70)$ \\
\hline Tertile3 & $18 / 62$ & I.39 $(0.74,2.58)$ & I. $18(0.57,2.48)$ & I.44 $(0.63,3.30)$ \\
\hline$P_{\text {trend }}$ & & 0.349 & 0.734 & 0.431 \\
\hline Per I SD increase & & $1.05(0.87,1.27)$ & $0.99(0.80,1.22)$ & I.07 $(0.78, I .45)$ \\
\hline \multicolumn{5}{|l|}{ Betaine } \\
\hline Tertile I & $27 / 62$ & 1.00 (ref.) & 1.00 (ref.) & 1.00 (ref.) \\
\hline Tertile2 & $16 / 63$ & $0.58(0.35,0.97)$ & $0.61(0.37,1.00)$ & $0.54(0.28,1.06)$ \\
\hline Tertile3 & $14 / 62$ & $0.52(0.30,0.89)$ & $0.52(0.30,0.88)$ & $0.4 I(0.19,0.86)$ \\
\hline$P_{\text {trend }}$ & & 0.011 & 0.010 & 0.015 \\
\hline Per I SD increase & & $0.80(0.64,0.99)$ & $0.79(0.62,1.00)$ & $0.68(0.47,1.00)$ \\
\hline \multicolumn{5}{|l|}{ DMG } \\
\hline Tertile I & $16 / 62$ & 1.00 (ref.) & 1.00 (ref.) & 1.00 (ref.) \\
\hline Tertile2 & $20 / 62$ & $1.25(0.72,2.18)$ & I.3I $(0.75,2.29)$ & I.63 $(0.79,3.39)$ \\
\hline Tertile3 & $21 / 63$ & $1.29(0.75,2.23)$ & I.32 $(0.77,2.27)$ & $1.32(0.65,2.64)$ \\
\hline$P_{\text {trend }}$ & & 0.366 & 0.321 & 0.189 \\
\hline Per I SD increase & & I.II $(0.93$, I.33) & $1.05(0.88,1.26)$ & $1.02(0.86,1.21)$ \\
\hline \multicolumn{5}{|l|}{ Methionine } \\
\hline Tertile I & $18 / 62$ & 1.00 (ref.) & 1.00 (ref.) & 1.00 (ref.) \\
\hline Tertile2 & $20 / 63$ & $1.09(0.64,1.86)$ & $1.15(0.69,1.93)$ & $0.97(0.50,1.89)$ \\
\hline Tertile3 & $19 / 62$ & $1.06(0.62,1.81)$ & $1.06(0.62,1.80)$ & $1.10(0.56,2.14)$ \\
\hline$P_{\text {trend }}$ & & 0.846 & 0.835 & 0.911 \\
\hline Per I SD increase & & $0.95(0.76,1.19)$ & $0.95(0.77,1.17)$ & $0.92(0.69,1.22)$ \\
\hline \multicolumn{5}{|l|}{ TMAO } \\
\hline Tertile I & $18 / 62$ & 1.00 (ref.) & $\mathrm{I} .00$ (ref.) & 1.00 (ref.) \\
\hline Tertile2 & $24 / 62$ & $1.33(0.81,2.20)$ & $1.38(0.85,2.24)$ & $1.23(0.66,2.29)$ \\
\hline Tertile3 & $15 / 63$ & $0.82(0.46,1.48)$ & $0.85(0.47,1.52)$ & $0.74(0.37, \mathrm{I} .49)$ \\
\hline$P_{\text {trend }}$ & & 0.533 & 0.617 & 0.421 \\
\hline Per I SD increase & & $1.00(0.79,1.25)$ & $1.02(0.82,1.26)$ & I.02 (0.78, I.33) \\
\hline
\end{tabular}

Notes: ${ }^{2}$ Model I: adjusted for gestational age at blood sampling, pre-pregnancy BMI, age, parity, mode of conception, ethnicity, occupation, infant sex, hypertensive disorders in pregnancy, gestational weight gain. Model 2: adjusted for covariates in model I plus mutually adjusted five plasma metabolites.

Abbreviations: DMG, dimethylglycine, TMAO, trimethylamine $\mathrm{N}$-oxide; $95 \% \mathrm{Cl}, 95 \%$ confidence interval; RR, relative risk.

examining plasma betaine in relation to T2DM risk; the results were consistent. ${ }^{25-29}$ Two large prospective cohort studies and two nested case-control studies, conducted in Norway, ${ }^{28}$ Netherlands, ${ }^{25}$ Spain $^{26}$ and United States, ${ }^{29}$ respectively, consistently showed an inverse relation between plasma betaine and T2DM risk. Besides, a crosssectional study of older adults in the United States also showed that subjects with higher levels of betaine had lower odds of T2DM. ${ }^{27}$ The consistent result from different ethnic groups or different study designs, suggesting that plasma betaine have a strong relationship with T2D or GDM.

After all, the mechanism and causality were still uncertain since our study was an observational study. Betaine has been reported to improve pathological processes related to GDM. Betaine supplementation to mice with nonalcoholic fatty liver reverses hepatic insulin resistance by increasing the activation of insulin receptor substrate 1 (IRS1). ${ }^{16}$ In mouse models, betaine supplementation improved glucose homeostasis via increasing the levels 
Table 3 Associations of Metabolites Ratios with GDM Risk ${ }^{\mathrm{a}}$

\begin{tabular}{|l|c|c|c|}
\hline & GDM/ & Unadjusted & Adjusted $^{\mathbf{a}}$ \\
\cline { 3 - 4 } & Subtotal & RR (95\% CI) & RR (95\% CI) \\
\hline Betaine: & & & \\
choline & & & \\
ratio & & & \\
Tertilel & $24 / 63$ & 1.00 (ref.) & 1.00 (ref.) \\
Tertile2 & $23 / 61$ & $0.99(0.63,1.55)$ & $1.17(0.74,1.85)$ \\
Tertile3 & $10 / 63$ & $0.42(0.22,0.80)$ & $0.48(0.25,0.91)$ \\
$P_{\text {trend }}$ & & 0.010 & 0.031 \\
\hline DMG: & & & \\
betine & & & \\
ratio & & & $1.00($ ref.) \\
Tertile3 & $29 / 62$ & $1.00($ ref.) & $0.54(0.32,0.90)$ \\
Tertile2 & $16 / 63$ & $0.54(0.33,0.90)$ & $0.42(0.24,0.74)$ \\
Tertile & $13 / 62$ & $0.42(0.23,0.73)$ & 0.001 \\
$P_{\text {trend }}$ & & 0.001 & \\
\hline
\end{tabular}

Notes: adjusted for gestational age at blood sampling, pre-pregnancy BMI, age, parity, use of ART, ethnicity, occupation, infant sex, hypertensive disorders in pregnancy, gestational weight gain.

Abbreviations: DMG, dimethylglycine; $95 \% \mathrm{Cl}, 95 \%$ confidence interval; RR, relative risk.

of hepatic and circulating fibroblast growth factor 21 (FGF21), a hormone is secreted by the liver and regulates whole-body glucose and lipid metabolism. ${ }^{14}$ Also, betainemodulated improvements in metabolic health may also involve its osmolyte functions and the reduction of oxidative stress. ${ }^{14,30}$

We noted a significant inverse association between betaine: choline ratio and GDM risk in the present study. The ratios of product: substrate may reflect the activities of involved enzymes. Choline can be synthesized through the hepatic phosphatidylethanolamine N-methyltransferase (PEMT). Enhanced hepatic PEMT activity would increase choline, while depleting betaine, a source for the PEMT

Table 4 AUC and the Comparison ${ }^{\mathrm{a}}$

\begin{tabular}{|l|c|c|c|}
\hline & AUC & $95 \% \mathbf{~ C l}$ & $P$ \\
\hline Curvel & 0.67 & $0.60,0.74$ & ref. \\
Curve2 & 0.74 & $0.67,0.80$ & 0.067 \\
Curve3 & 0.76 & $0.69,0.82$ & 0.042 \\
Curve4 & 0.79 & $0.72,0.84$ & 0.006 \\
\hline
\end{tabular}

Notes: a Curve I represents the basic risk factors (gestational age at blood sampling, pre-pregnancy BMI, age, parity, use of ART, ethnicity, occupation, infant sex, hypertensive disorders in pregnancy, gestational weight gain). Curve 2 represents basic factors plus choline, methionine, DMG and TMAO. Curve 3 indicates covariates in Curve 2 plus betaine. Curve 4 indicates basic risk factors, DMG: betaine, betaine: choline, TMAO, methionine and DMG.

Abbreviations: $95 \% \mathrm{Cl}, 95 \%$ confidence interval; $\mathrm{AUC}$, area under the curve. reaction, resulting in a lower betaine: choline ratio. ${ }^{21}$ This result was supported by an animal study in which lack of PEMT $\left(\mathrm{Pemt}^{-1}\right)$ showed a protective effect against dietinduced obesity and insulin resistance. ${ }^{31}$ Betaine can be catalyzed by betaine-homocysteine methyltransferase (BHMT) to form methionine while generate DMG. Enhanced BHMT activity would decrease plasma betaine while increase plasma DMG. Our observed protective role of lower DMG: betaine ratio, suggesting less active of BHMT, in GDM was strengthened by a study which showed Bhmt deletion resulted in enhanced insulin sensitivity and glucose tolerance. ${ }^{32}$ Besides, ratios of metabolites performed better in predicting GDM risk than metabolites alone in our study. These results suggest that activities of enzymes involve betaine metabolism might also contribute to the pathogenesis of GDM in women with twin pregnancy.

TMAO is a metabolite derived from L-carnitine, choline, and betaine by gut microbiota. An increasing number of epidemiologic studies have investigated the associations of TMAO with the risk of GDM or T2DM. Most of previous studies, ${ }^{28,33,34}$ but not all, ${ }^{26}$ reported a positive association between levels of TMAO and T2DM risk. As for GDM, two studies showed that GDM group had lower levels of plasma TMAO, ${ }^{17,18}$ while another study from

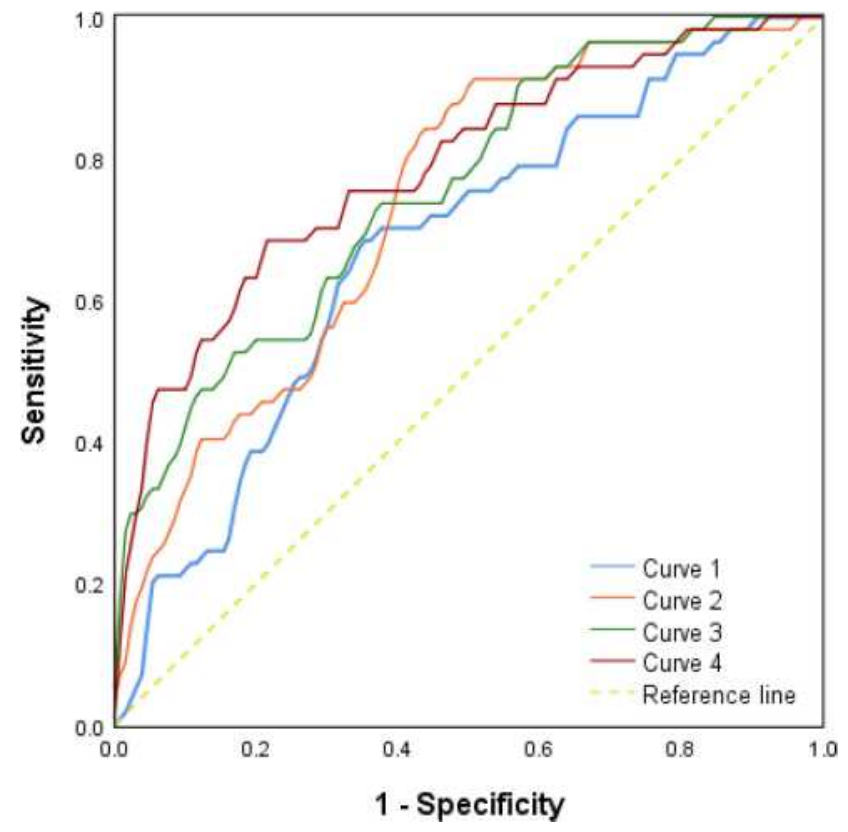

Figure 2 ROC curve analysis. Curve I (blue) represents the basic risk factors (Table 2: covariates in model I). Curve 2 (orange) represents basic factors plus choline, methionine, DMG and TMAO. Curve 3 (green) indicates covariates in Curve 2 plus betaine. Curve 4 (red) indicates basic risk factors, DMG: betaine, betaine: choline, TMAO, methionine and DMG. 
China observed inverse results. ${ }^{19} \mathrm{~A}$ U-shaped association of choline status in early pregnancy with GDM risk were reported in a nested case-control study in China. ${ }^{18}$ Additionally, a recent metabonomic study in Finland observed an increased risk of T2DM in relation to DMG. ${ }^{35}$ However, in our study, TMAO, choline, DMG or methionine was not associated with GDM risk. Differences in characteristics and disease status of population across studies might partly explain such inconsistency. Also, these metabolites may have differential associations with GDM among women with singleton or twin pregnancy. In addition, a fluctuation in the levels of one metabolite may alter the levels of other metabolites because they share the same metabolic pathway. However, mutual adjustments of these metabolites were not conducted in previous studies when assessing their relations with GDM or T2DM.

The main strength of our study was the prospective design. The relatively long follow-up time between the blood sampling and GDM diagnosis (median: 8.1 weeks, 90\% range: 5.4-11.4) might reduce the possibility of reverse causation. Extensive collections and full adjustments of multiple risk factors and related metabolites were also considered as strengths of this study. However, there were some limitations in our study. First, small sample size limits the power of our study. Second, only one time point assessment may not represent the metabolites status during whole pregnancy. Also, our participants were recruited in a large tertiary referral hospital and 69\% of them are white collar workers, which may decrease the generalizability of our results.

\section{Conclusion}

Higher levels of plasma betaine in the second trimester of pregnancy were associated with lower GDM risk among Chinese women with twin pregnancy. Moreover, the well performance of the ratio of betaine: choline and DMG: betaine in predicting GDM risk is worthy of attention. Our results need to be confirmed in larger cohorts of twin gestations.

\section{Ethics Approval and Informed Consent}

This study was conducted in accordance with the Declaration of Helsinki and approved by the Peking University Third Hospital Medical Ethics Committee (IRB00006761-2016145), and all participants provided informed consent.

\section{Acknowledgments}

We thank all the participants who took part in our study and the clinicians, nurses and laboratory technicians who collected samples and performed the tests. This work was supported by the National Key R\&D Program of China (2018YFC1002900), and National Key R\&D Program of China (2016YFC1000400).

\section{Author Contributions}

All authors made contributions to conception and design, acquisition of data, or data analysis, and took part in drafting the article or revising it critically, have agreed to submit to the current journal, gave final approval of the version to be published, and agreed to be accountable for all aspects of the work.

\section{Funding}

This work was supported by the National Key R\&D Program of China (2018YFC1002900), and National Key R\&D Program of China (2016YFC1000400).

\section{Disclosure}

The authors have no conflicts of interest to disclose.

\section{References}

1. Guariguata L, Linnenkamp U, Beagley J, Whiting DR, Cho NH. Global estimates of the prevalence of hyperglycaemia in pregnancy. Diabetes Res Clin Pract. 2014;103(2):176-185. doi:10.1016/j. diabres.2013.11.003.

2. Xu Q, Gao ZY, Li LM, et al. The Association of Maternal Body Composition and Dietary Intake with the Risk of Gestational Diabetes Mellitus during the Second Trimester in a Cohort of Chinese Pregnant Women. Biomed Environ Sci. 2016;29(1):1-11. doi:10.3967/bes2016.001.

3. Kahveci B, Melekoglu R, Evruke IC, Cetin C. The effect of advanced maternal age on perinatal outcomes in nulliparous singleton pregnancies. BMC Pregnancy Childbirth. 2018;18(1):343. doi:10.1186/s12884-018-1984-x.

4. Du HY, Jiang H, Chen B, et al. Association of Dietary Pattern during Pregnancy and Gestational Diabetes Mellitus: a Prospective Cohort Study in Northern China. Biomed Environ Sci. 2017;30(12):887-897. doi:10.3967/bes2017.119.

5. Fetita LS, Sobngwi E, Serradas P, Calvo F, Gautier JF. Consequences of fetal exposure to maternal diabetes in offspring. J Clin Endocrinol Metab. 2006;91(10):3718-3724. doi:10.1210/jc.2006-0624.

6. Silverman BL, Rizzo TA, Cho NH, Metzger BE. Long-term effects of the intrauterine environment: the Northwestern University diabetes in pregnancy center. Diabetes Care. 1998;21(SUPPL.2):B142-B149.

7. Chang $\mathrm{Y}$, Chen $\mathrm{X}$, Zhang ZK. Intrauterine exposure to maternal diabetes is associated with adiposity in children at 6 years of age in China. Biomed Environ Sci. 2015;28(2):140-142. doi:10.3967/ bes2015.017.

8. Retnakaran R, Shah BR. Impact of twin gestation and fetal sex on maternal risk of diabetes during and after pregnancy. Diabetes Care. 2016;39(8):e110-111. doi:10.2337/dc16-0825. 
9. Hiersch L, Berger H, Okby R, et al. Gestational diabetes mellitus is associated with adverse outcomes in twin pregnancies. Am J Obstet Gynecol. 2019;220(1):102.e101-102.e108. doi:10.1016/j. ajog.2018.10.027.

10. Hung TH, Hsieh TT, Shaw SW, Kok Seong C, Chen SF. Risk factors and adverse maternal and perinatal outcomes for women with dichorionic twin pregnancies complicated by gestational diabetes mellitus: a retrospective cross-sectional study. J Diabetes Investig. 2020. doi:10.1111/jdi.13441.

11. Lai FY, Johnson JA, Dover D, Kaul P. Outcomes of singleton and twin pregnancies complicated by pre-existing diabetes and gestational diabetes: a population-based study in Alberta, Canada, 2005-11. J Diabetes. 2016;8(1):45-55. doi:10.1111/17530407.12255 .

12. Zeisel SH. Choline: critical role during fetal development and dietary requirements in adults. Annu Rev Nutr. 2006;26:229-250. doi:10.1146/annurev.nutr.26.061505.111156

13. Ueland PM. Choline and betaine in health and disease. J Inherit Metab Dis. 2011;34(1):3-15. doi:10.1007/s10545-010-9088-4.

14. Ejaz A, Martinez-Guino L, Goldfine AB, et al. Dietary betaine supplementation increases Fgf21 levels to improve glucose homeostasis and reduce hepatic lipid accumulation in mice. Diabetes. 2016;65(4):902-912. doi:10.2337/db15-1094.

15. Gao X, Liu X, Xu J, Xue C, Xue Y, Wang Y. Dietary trimethylamine $\mathrm{N}$-oxide exacerbates impaired glucose tolerance in mice fed a high fat diet. J Biosci Bioeng. 2014;118(4):476-481. doi:10.1016/j. jbiosc.2014.03.001.

16. Kathirvel E, Morgan K, Nandgiri G, et al. Betaine improves nonalcoholic fatty liver and associated hepatic insulin resistance: a potential mechanism for hepatoprotection by betaine. Am J Physiol Gastrointest Liver Physiol. 2010;299(5):G1068-1077. doi:10.1152/ ajpgi.00249.2010.

17. Diaz SO, Pinto J, Graca G, et al. Metabolic biomarkers of prenatal disorders: an exploratory NMR metabonomics study of second trimester maternal urine and blood plasma. J Proteome Res. 2011;10 (8):3732-3742. doi:10.1021/pr200352m.

18. Huo X, Li J, Cao YF, et al. Trimethylamine N-oxide metabolites in early pregnancy and risk of gestational diabetes: a Nested Case-Control Study. J Clin Endocrinol Metab. 2019;104 (11):5529-5539. doi:10.1210/jc.2019-00710.

19. Li P, Zhong $\mathrm{C}$, $\mathrm{Li} \mathrm{S}$, et al. Plasma concentration of trimethylamine-N-oxide and risk of gestational diabetes mellitus. Am J Clin Nutr. 2018;108(3):603-610. doi:10.1093/ajcn/nqy116.

20. Santana DS, Silveira C, Costa ML, et al. Perinatal outcomes in twin pregnancies complicated by maternal morbidity: evidence from the WHO multicountry survey on maternal and newborn health. BMC Pregnancy Childbirth. 2018;18(1):449. doi:10.1186/s12884-0182082-9.

21. Yan J, Winter LB, Burns-Whitmore B, Vermeylen F, Caudill MA. Plasma choline metabolites associate with metabolic stress among young overweight men in a genotype-specific manner. Nutr Diabetes. 2012;2(10):e49. doi:10.1038/nutd.2012.23.

22. Metzger BE, Gabbe SG, Persson B, et al.; International Association of, D., Pregnancy Study Groups Consensus, P. International association of diabetes and pregnancy study groups recommendations on the diagnosis and classification of hyperglycemia in pregnancy. Diabetes Care. 2010;33(3):676-682. doi:10.2337/dc09-1848.
23. Barros AJ, Hirakata VN. Alternatives for logistic regression in cross-sectional studies: an empirical comparison of models that directly estimate the prevalence ratio. BMC Med Res Methodol. 2003;3:21. doi:10.1186/1471-2288-3-21.

24. Vounzoulaki E, Khunti K, Abner SC, Tan BK, Davies MJ, Gillies CL. Progression to type 2 diabetes in women with a known history of gestational diabetes: systematic review and meta-analysis. BMJ (Clinical Research Ed). 2020;369:m1361. doi:10.1136/bmj.m1361.

25. Garcia E, Oste MCJ, Bennett DW, et al. High betaine, a trimethylamine $\mathrm{N}$-oxide related metabolite, is prospectively associated with low future risk of type 2 Diabetes Mellitus in the PREVEND Study. J Clin Med. 2019;8(11):1813. doi:10.3390/ jem8111813.

26. Papandreou C, Bulló M, Zheng Y, et al. Plasma trimethylamine$\mathrm{N}$-oxide and related metabolites are associated with type 2 diabetes risk in the Prevención con Dieta Mediterránea (PREDIMED) trial. Am J Clin Nutr. 2018;108(1):163-173. doi:10.1093/ajen/nqy058.

27. Roe AJ, Zhang S, Bhadelia RA, et al. Choline and its metabolites are differently associated with cardiometabolic risk factors, history of cardiovascular disease, and MRI-documented cerebrovascular disease in older adults. Am J Clin Nutr. 2017;105(6):1283-1290. doi:10.3945/ajen.116.137158.

28. Svingen GF, Schartum-Hansen H, Pedersen ER, et al. Prospective associations of systemic and urinary choline metabolites with incident type 2 diabetes. Clin Chem. 2016;62(5):755-765. doi:10.1373/ clinchem.2015.250761.

29. Walford GA, Ma Y, Clish C, Florez JC, Wang TJ, Gerszten RE. Metabolite profiles of diabetes incidence and intervention response in the diabetes prevention program. Diabetes. 2016;65(5):1424-1433. doi: $10.2337 / \mathrm{db} 15-1063$

30. Jung YS, Kim SJ, Kwon DY, et al. Alleviation of alcoholic liver injury by betaine involves an enhancement of antioxidant defense via regulation of sulfur amino acid metabolism. Food Chem Toxicol. 2013;62:292-298. doi:10.1016/j.fct.2013.08.049.

31. Jacobs RL, Zhao Y, Koonen DP, et al. Impaired de novo choline synthesis explains why phosphatidylethanolamine $\mathrm{N}$-methyltransferase-deficient mice are protected from diet-induced obesity. J Biol Chem. 2010;285(29):22403-22413. doi:10.1074/jbc. M110.108514.

32. Teng YW, Ellis JM, Coleman RA, Zeisel SH. Mouse betaine-homocysteine S-methyltransferase deficiency reduces body fat via increasing energy expenditure and impairing lipid synthesis and enhancing glucose oxidation in white adipose tissue. $\mathrm{J} \mathrm{Biol}$ Chem. 2012;287(20):16187-16198. doi:10.1074/jbc.M111.303255.

33. Obeid R, Awwad HM, Rabagny Y, Graeber S, Herrmann W, Geisel J. Plasma trimethylamine N-oxide concentration is associated with choline, phospholipids, and methyl metabolism. Am J Clin Nutr. 2016;103(3):703-711. doi:10.3945/ajcn.115.121269.

34. Shan Z, Sun T, Huang $H$, et al. Association between microbiota-dependent metabolite trimethylamine-N-oxide and type 2 diabetes. Am J Clin Nutr. 2017;106(3):888-894. doi:10.3945/ ajcn.117.157107.

35. Vangipurapu J, Fernandes Silva L, Kuulasmaa T, Smith U, Laakso M. Microbiota-related metabolites and the risk of type 2 diabetes. Diabetes Care. 2020;43(6):1319-1325. doi:10.2337/dc19-2533. 


\section{Publish your work in this journal}

Diabetes, Metabolic Syndrome and Obesity: Targets and Therapy is an international, peer-reviewed open-access journal committed to the rapid publication of the latest laboratory and clinical findings in the fields of diabetes, metabolic syndrome and obesity research. Original research, review, case reports, hypothesis formation, expert opinion and commentaries are all considered for publication. The manuscript management system is completely online and includes a very quick and fair peer-review system, which is all easy to use. Visit http://www.dovepress.com/testimonials.php to read real quotes from published authors. 